\title{
Efficient Furfuryl Alcohol Synthesis from Furfural over Magnetically Recoverable Catalysts: Does the Catalyst Stabilizing Medium Matter?
}

\author{
Kenan Alibegovic ${ }^{[a]}$, David Gene Morgan ${ }^{[a]}$, Yaroslav Losovyj] ${ }^{[a]}$ Maren Pink ${ }^{[a]}$, Barry D. Stein ${ }^{[b]}$, Nina V. \\ Kuchkina ${ }^{[c]}$, Elena S. Serkova, ${ }^{[c]}$ Kseniya E. Salnikova, ${ }^{[d]}$ Zinaida B. Shifrina, ${ }^{[c]}$ Valentina G. Matveeva, ${ }^{*}$ \\ [d,e] Esther M. Sulman, ${ }^{[d]}$ Lyudmila M. Bronstein*[a, c, f]
}

\begin{abstract}
We report hydrogenation of furfural (FF) to furfuryl alcohol (FA) with novel Pt-and Pd-containing magnetite nanoparticles (NPs) stabilized by polyphenylquinoxaline (PPQ) and hyperbranched pyridylphenylene polymer (PPP). FF is one of the major ingredients of biooil produced by biomass pyrolysis, while FA is a source of valueadded chemicals, thus, creating a sustainable path from biomass to important compounds. We demonstrate that catalytic NPs $\left(\mathrm{Pt}^{0}\right.$ or $\left.\mathrm{Pd}^{0}\right)$ of approximately $3 \mathrm{~nm}$ in diameter form in the polymer shells of magnetite NPs and the catalysts are magnetically recoverable. The search for optimal reaction conditions of the FF hydrogenation revealed
\end{abstract}

\section{Introduction}

Obtaining value-added chemicals from biomass and biooil instead of petrochemical sources received considerable attention due to limited amounts of fossil fuels. Furfural (FF) is one of the major ingredients of biooil produced by biomass pyrolysis and a starting compound for a number of useful chemicals including furfuryl alcohol (FA). FF can be also formed by the acid-catalyzed dehydration of $\mathrm{C} 5$ and $\mathrm{C} 6$ carbohydrates in the biomass such as hemicellulose. ${ }^{[1]} \mathrm{FA}$ is acquired by FF hydrogenation along with other products such as furan, tetrahydrofuran, tetrahydrofurfural, and tetrahydrofurfuryl alcohol which can be used as plasticizers and intermediates in pharmaceuticals syntheses. ${ }^{[2]}$ Scheme 1 shows the complexity of the process which could lead to multiple products.

FA is a valuable compound employed for syntheses of polyfurfuryl alcohol nanocomposites without the use of solvents or surfactants and for foundry binders. ${ }^{[3]}$ Various noble and transition metals such as Pd, Pt, Ru, Ni, Cu and also bimetallic Pd-Ni, Pd-Ir, Pd-Ru, Pt-Sn catalysts have been tested in the FF hydrogenation to $F A .{ }^{[4]}$

[a] K. Alibegovic, Dr. D. G. Morgan, Dr. Y. Losovyj, Dr. M. Pink, Dr. L. M. Bronstein

Department of Chemistry, Indiana University, Bloomington, IN 47405, USA

E-mail: Iybronst@indiana.edu

[b] Dr. Barry D. Stein

Department of Biology, Indiana University, Bloomington, IN 47405, USA

[c] Dr. N. V. Kuchkina, E. S. Serkova, Prof. Zinaida B. Shifrina, Dr. L. M. Bronstein

A.N. Nesmeyanov Institute of Organoelement Compounds, Russian Academy of Sciences, 28 Vavilov St., Moscow, 119991 Russia

[d] K. E. Salnikova, Prof. V. G. Matveeva, Prof. E. M. Sulman Department of Biotechnology and Chemistry

Tver State Technical University, 22 A. Nikitina St., Tver, 170026 Russia

[e] Prof. V. G. Matveeva

Tver State University, Regional Technological Center, 33

Zhelyabova St., Tver, 170100, Russia. E-mail:

matveeva@science.tver.ru

[f] Dr. L. M. Bronstein,

Department of Physics, Faculty of Science,

King Abdulaziz University,Jeddah, Saudi Arabia

Supporting information for this article can be found under: that the highest selectivity is obtained at $120^{\circ} \mathrm{C}$ and $6 \mathrm{MPa}$ hydrogen pressure in $i$-propanol as solvent. The solvent effect is due to combination of good FF solubility and accessibility of catalytic NPs for the FF adsorption. A comparison of the catalytic activities of the Pdcontaining magnetite NPs stabilized by PPQ and PPP validates the advantages of the open and rigid structure of the hyperbranched PPP vs. linear PPQ. For Pd-containing magnetite NPs stabilized by PPP, the high selectivity to FA of $99.3 \%$ at nearly $100 \%$ FF conversion was achieved at a remarkable activity of $871 \mathrm{~min}^{-1}$ and high catalyst stability.

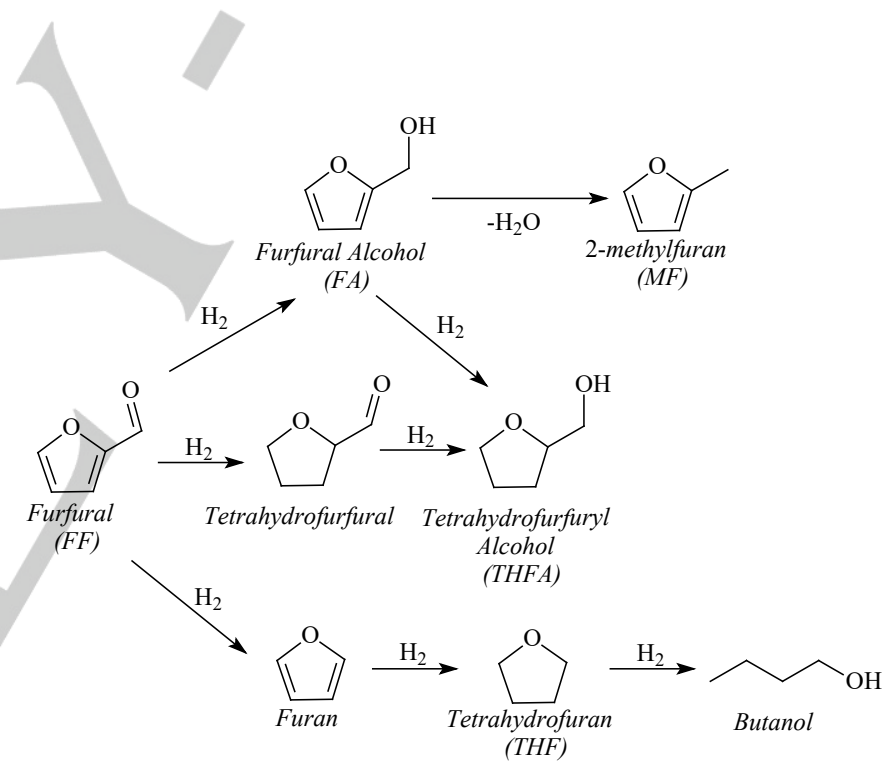

Scheme 1. The possible routes of FF hydrogenation.

Magnetically recoverable catalysts are widely used in numerous catalytic reactions to allow for easy magnetic separation in batch reactions or stabilization of a catalytic layer in flow processes. ${ }^{[5]}$ To the best of our knowledge, there is only a single example of using magnetic $\mathrm{Fe}(\mathrm{NiFe}) \mathrm{O}_{4}-\mathrm{SiO}_{2}$ nanocatalyst for $\mathrm{FF}$ hydrogenation. ${ }^{[6]}$ This catalyst demonstrated high efficiency and selectivity to FA but exposure of the $\mathrm{Fe}(\mathrm{NiFe}) \mathrm{O}_{4}$ nanoparticles (NPs) in the silica network does not warrant their stability in the long run despite easy recoverability due to magnetic separation.

Better stabilization of catalytic and magnetic species can be carried out using a polymer as stabilizer. In our preceding work thermally stable polyphenylquinoxaline $(\mathrm{PPQ})^{[7]}$ and a hyperbranched pyridylphenylene polymer (PPP),${ }^{[8]}$ whose structures are presented in Scheme 2, were employed as efficient stabilizers of $\mathrm{Zn}$ containing magnetic oxides designed for a syngas-to-methanol 
transformation. ${ }^{[9]}$ Both polymers are thermally stable up to 400$500^{\circ} \mathrm{C}$, allowing for a wide temperature range of catalytic reactions. The presence of nitrogen-containing heterocycles in their repeating units allows for coordination with various metals. It is worth noting that $P P Q$ is a linear polymer, thus may form a comparatively dense shell on the NP surface. Alternatively, a hyperbranched, open and rigid structure of PPP may allow for better access to catalytic species.

Here, we demonstrate that the PPQ or PPP shells of iron oxide NPs successfully stabilize formation of well-defined Pt or Pd NPs in a one-pot reaction without isolation and purification of intermediates. The availability of the catalytic NPs for furfural and their efficient modification/stabilization with polymers allow for high selectivity in FA formation as well as high activity and stability after magnetic recovery.
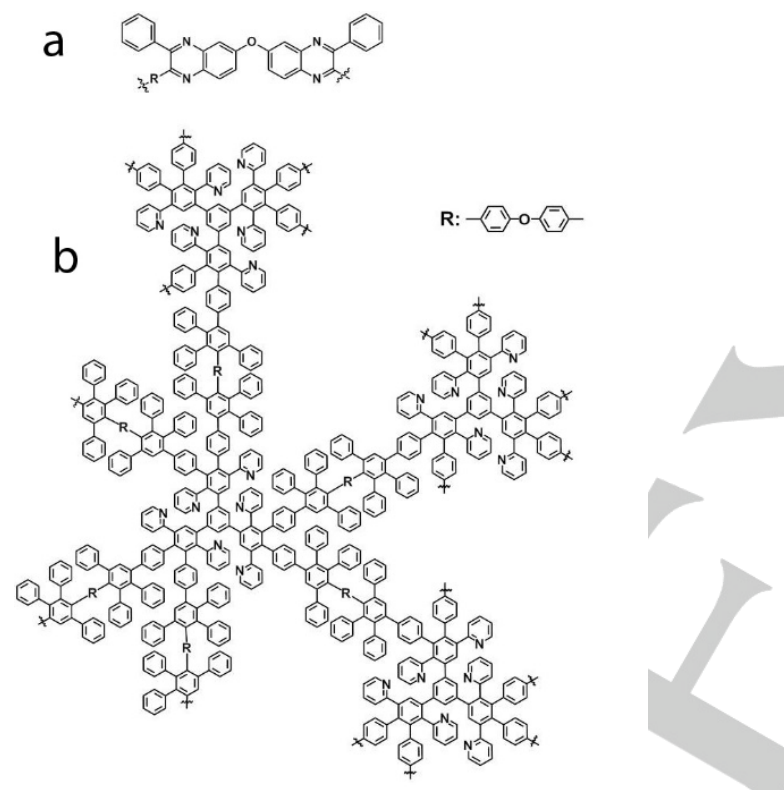

Scheme 2. The structures of the PPQ (a) and PPP (b) repeating units.

\section{Results and Discussion}

Recently, a sustainable chemistry effort has been focused on several key interconnected areas: (i) replacement of fossil fuels with renewable sources for obtaining value-added chemicals, (ii) development of exceptionally efficient and stable catalysts for these processes, and (iii) minimization of the reaction steps in preparation of these catalysts. Therefore, a preferable pathway for syntheses of magnetically recoverable catalysts designed for FF hydrogenation is a one-pot reaction to avoid isolation and purification of intermediates. On the other hand, the catalytic species need to be placed on top of magnetic support NPs for access to reagents. To meet both requirements, we synthesized iron oxide NPs by thermal decomposition of $\mathrm{Fe}(\mathrm{acac})_{3}$ in boiling benzyl ether and then injected the $\mathrm{Pt}(\mathrm{acac})_{2}$ or $\mathrm{Pd}(\mathrm{acac})_{2}$ solution into the hot reaction solution containing preformed iron oxide NPs.
The magnetic separation of the catalyst is shown in Figure S1 (the Supporting Information, SI). The control experiment carried out without injection of the $\mathrm{Pt}$ or $\mathrm{Pd}$ precursor shows that particles formed include multicore NPs with a mean size of about $21 \mathrm{~nm}$ and a smaller fraction of quasi-spherical single-core NPs with diameters in the range of $6-10 \mathrm{~nm}^{\left[{ }^{[10]}\right.}$ The structure of both types of NPs is that of magnetite $\left(\mathrm{Fe}_{3} \mathrm{O}_{4}\right)$ as was confirmed by a combination of XRD and XPS. ${ }^{[10]}$ See also Figure S2 (SI) for the representative TEM image and XRD pattern. When $\mathrm{Pt}(\mathrm{acac})_{2}$ or $\mathrm{Pd}(\mathrm{acac})_{2}$ is injected into the reaction solution at the reaction temperature, two scenarios are possible. The $\mathrm{Pt}$ or $\mathrm{Pd}$ precursor can be rapidly decomposed and nucleate without coordinating with the magnetite NP polymer shell, leading to catalytic NPs located between magnetite NPs. In the other scenario, $\mathrm{Pt}(\mathrm{Pd})$ species (nuclei) first coordinate with nitrogen-containing heterocycles followed by a catalytic NP growth in the $\mathrm{Fe}_{3} \mathrm{O}_{4} \mathrm{NP}$ polymer shells. In the former case, the catalytic NPs would be unsupported by magnetite NPs, i.e., not magnetically recoverable, thus making it an undesirable outcome.

\section{Structure of Pt- and Pd-containing magnetite NPs}

Figure 1 shows TEM images of four Pt-containing magnetite NPs prepared with different amounts of $\mathrm{Pt}(\mathrm{acac})_{2}$. In addition to multicore and single core iron oxide NPs, the images contain darker dots (indicated by red arrows) which are indicative of Pt-containing NPs with a higher electron contrast. In the samples prepared with 0.10 and $0.05 \mathrm{mmol}$ of $\mathrm{Pt}(\mathrm{acac})_{2}$ (Pt-1 and Pt-2, Table 1, respectively) the Pt-containing NPs are located both in vicinity and between magnetite NPs, revealing that some $\mathrm{Pt}$ and $\mathrm{Fe}_{3} \mathrm{O}_{4} \mathrm{NPs}$ might be unconnected to each other. In case of the samples prepared with 0.02 and $0.01 \mathrm{mmol}$ of $\mathrm{Pt}(\mathrm{acac})_{2}$ (Pt-3 and Pt-4, Table 1, respectively), the Pt NPs are mainly adjacent to magnetite NPs. To confirm this assumption, Pt-1 and Pt-3 were magnetically recovered from chloroform solutions, then redispersed in chloroform and imaged. The images presented in Figure S3 (SI) indicate that in the Pt-1 sample all Pt based NPs seen between iron oxide NPs were removed during magnetic separation, while the Pt3 sample appears unchanged.

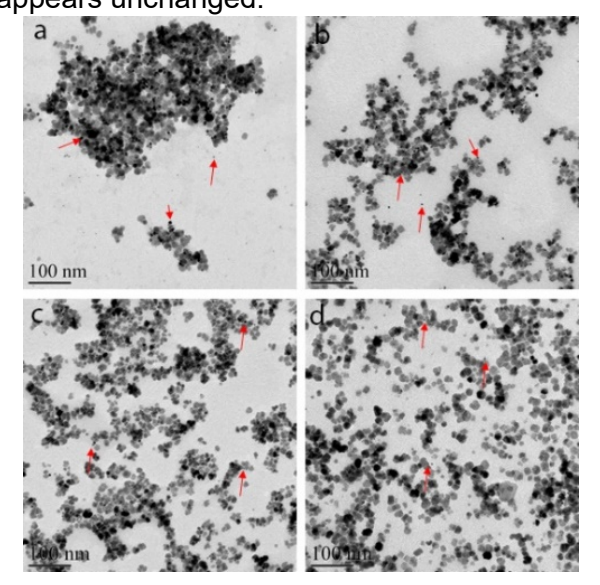

Figure 1. TEM images of Pt-containing iron oxide NPs prepared with different amounts of Pt(acac) 2: (a) $0.10 \mathrm{mmol}$ (Pt-1), (b) $0.05 \mathrm{mmol}$ (Pt-2), (c) $0.02 \mathrm{mmol}$ (Pt-3), and (d) $0.01 \mathrm{mmol}(\mathrm{Pt}-4)$. Red arrows indicate Pt-containing NPs. 
Table 1. Pt- and Pd-containing magnetite NPs stabilized by PPQ.

\begin{tabular}{lll} 
Sample notation & $\begin{array}{l}\left.\text { Pt(acac })_{2} \text { or Pd(acac }\right)_{2} \\
\text { amount, g (mmol) }\end{array}$ & $\begin{array}{l}\text { Pt or Pd NP size, } \\
\mathrm{nm}\end{array}$ \\
\hline Pt-1 & $0.0393(0.10)$ & $2.8 \pm 0.8$ \\
Pt-2 & $0.020(0.05)$ & $2.7 \pm 0.9$ \\
Pt-3 & $0.008(0.02)$ & $2.9 \pm 0.7$ \\
Pt-4 & $0.004(0.01)$ & $2.8 \pm 0.8$ \\
Pd-1 & $0.003(0.01)$ & $2.8 \pm 0.9$ \\
Pd-2 & $0.006(0.02)$ & $3.1 \pm 0.7$ \\
Pd-3 & $0.012(0.04)$ & $3.2 \pm 0.6$ \\
\hline
\end{tabular}

To confirm positioning of Pt vs $\mathrm{Fe}$ in the Pt-2 sample, we carried out STEM EDS imaging (Fig. 2). The mapping shows that the Ptcontaining NPs are in vicinity of iron oxide NPs.
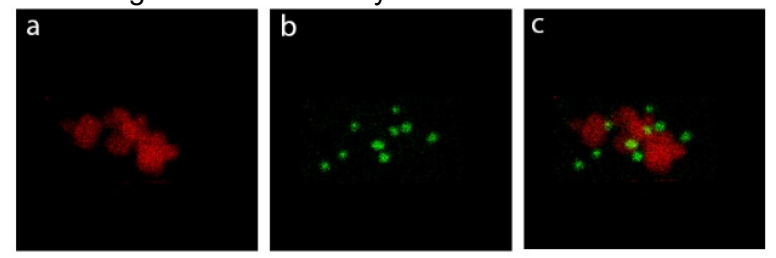

Figure 2. STEM EDS maps of Fe (a), Pt (b), and their superposition (c) for Pt-2.

For all $\mathrm{Pt}(\mathrm{acac})_{2}$ loadings, the Pt NP diameters are very similar and under $3 \mathrm{~nm}$. The XRD pattern of Pt-3 presented in Figure 3 shows the set of the Bragg reflections (black labeling in Fig. 3), the positions and intensity of which are typical for those of magnetite, ${ }^{[11]}$ similar to control magnetite NPs (Fig. S2, SI). The other set of reflections in Figure 3 labeled in blue, belongs to the $\mathrm{Pt}^{0}$ crystal structure. ${ }^{[12]}$

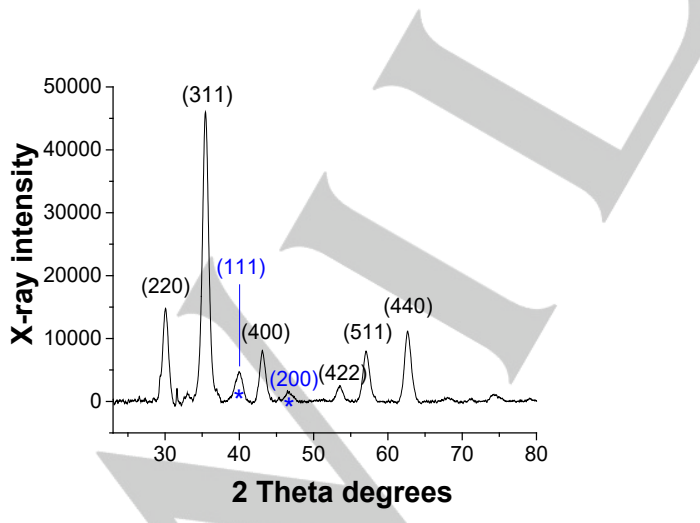

Figure 3. XRD pattern of Pt-3. Black labelling shows reflections of $\mathrm{Fe}_{3} \mathrm{O}_{4}$, while blue labelling is for $\mathrm{Pt}$.
The high resolution XPS spectra for Pt-2 in the Fe $2 p$ and Pt $4 f$ regions are presented in Figure 4. The HR Fe $2 p$ XPS spectrum shows a major peak with a binding energy $(\mathrm{BE})$ of $710.8 \mathrm{eV}$, which is characteristic of iron oxides. A satellite usually detected for $\mathrm{Fe}^{3+}$ ions at a BE value of 8-9 eV higher than the major peak, is absent. This satellite would indicate the excess of the $\mathrm{Fe}^{3+}$ species beyond the $\mathrm{Fe}^{3+}: \mathrm{Fe}^{2+}=2: 1$ ratio of magnetite. ${ }^{[13]}$ However, the combination of the $\mathrm{Fe}^{3+}$ and $\mathrm{Fe}^{2+}$ satellites leads to a flat plateau between the Fe $2 p 3 / 2$ and Fe $2 p 1 / 2$ peaks as is observed in our case. ${ }^{[14]}$ The HR Pt $4 \mathrm{f}$ XPS spectrum shows a major peak with a BE of $71.7 \mathrm{eV}$ which is also consistent with $\mathrm{Pt}^{0}$ of $\mathrm{Pt} \mathrm{NPs}{ }^{\left[{ }^{[15]}\right.}$ If only Pt and $\mathrm{Fe}$ are accounted for, the sample contains 92.4 at. \% of Fe and 7.6 at.\% of Pt.

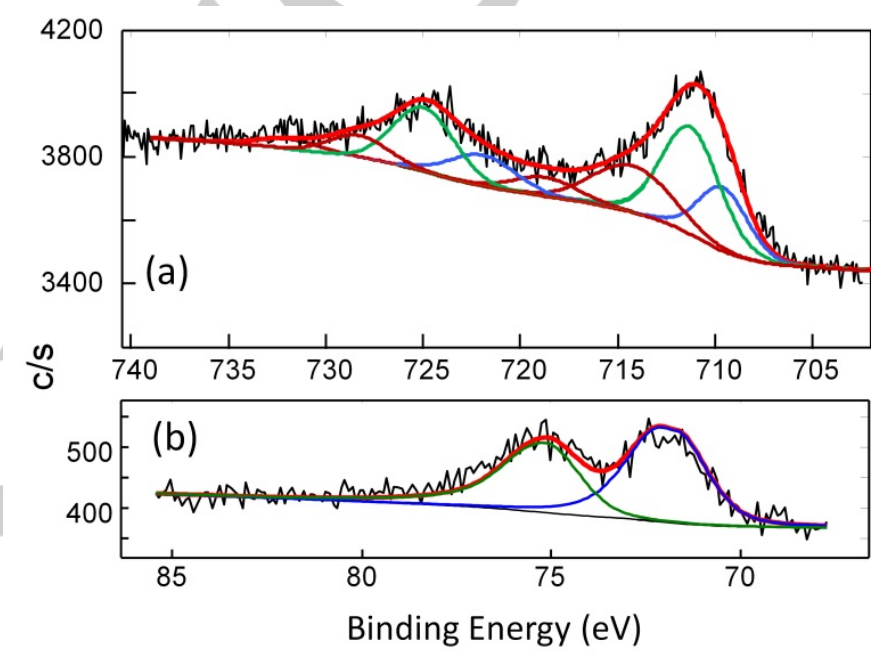

Figure 4. (a) HR Fe 2p XPS of Pt-2. Blue color represents $\mathrm{Fe}^{2+}$, green is for $\mathrm{Fe}^{3+}$, brown represents satellites, red is for generated fit, and black is for raw data. See Table S1 (SI) for the deconvolution parameters. (b) HR Pt 4f XPS of Pt-2. Blue and green lines represent $4 \mathrm{f} 3 / 2$ and $4 \mathrm{f} 1 / 2$ of $\mathrm{Pt}^{0}$, respectively. Red is for generated fit and black is for raw data.

Formation of $\mathrm{Pt}^{0} \mathrm{NPs}$ instead of $\mathrm{Pt}$ oxide which might be expected from the $\mathrm{Pt}(\mathrm{acac})_{2}$ thermal decomposition, is due to partial decomposition of benzyl ether into benzyl radicals, ${ }^{[16]}$ which, in turn, may serve as reducing agents. ${ }^{[17]}$

Pd-containing magnetite NPs stabilized by $P P Q$ were prepared at lower $\mathrm{Pd}(\mathrm{acac})_{2}$ loadings than those of $\mathrm{Pt}(\mathrm{acac})_{2}(0.01 \mathrm{mmol}$ for $\mathrm{Pd}-1,0.02 \mathrm{mmol}$ for Pd-2, and $0.04 \mathrm{mmol}$ for $\mathrm{Pd}-3$ ) to minimize the formation Pd NPs unconnected to magnetite NPs. The TEM images of these samples presented in Figure $\mathrm{S} 4$ (SI) show similar morphology as that observed for Pt-containing magnetite NPs. Moreover, even at the highest loading nearly all the Pd NPs are connected to magnetite NPs or located in their vicinity, allowing successful magnetic separation. The location of Pd and Fe species is confirmed by the STEM EDS maps presented in Figure 5 for Pd3. The XRD pattern of Pd-2 shows mainly reflections of magnetite and the (111) reflection of $\mathrm{Pd}^{0}$ (Fig. S5, SI). ${ }^{[18]}$ Appearance of only the strongest reflection was observed for other composite Pdcontaining materials. ${ }^{[19]}$ The XPS data are consistent with those of $X R D$ with the HR Fe $2 p$ XPS spectrum characteristic of solely 
$\mathrm{Fe}_{3} \mathrm{O}_{4}{ }^{[10,13]}$ (Fig. S6, SI) and the $\mathrm{HR} \mathrm{Pd} 3 \mathrm{~d}$ XPS spectrum representative of $\mathrm{Pd}^{0}$ (Fig. S7, SI). Thus, although FePd NPs are reported in literature, ${ }^{[20]}$ they were not formed in the reactions conditions described in this paper. Instead, $\mathrm{Pd}^{0} \mathrm{NPs}$ with diameters around $3 \mathrm{~nm}$ were made.
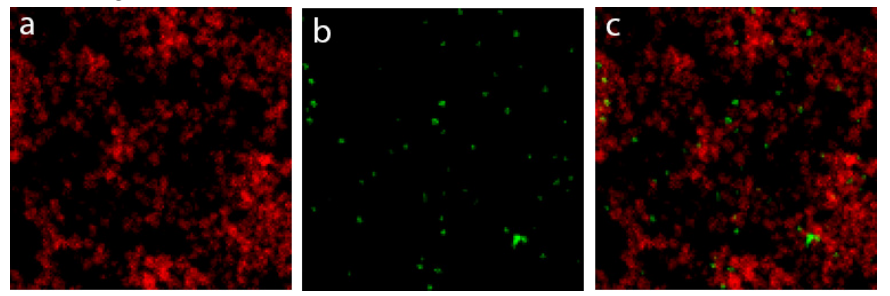

Figure 5. STEM EDS maps of Fe (a), Pd (b), and their superposition (c) for Pd-3.

Along with $P P Q, P d$-containing magnetite NPs were synthesized in the presence of PPP as capping molecules (Table 2). This was motivated by the results of our preceding work on doped $\mathrm{Zn}$ containing magnetic oxides NPs, where hyperbranched PPP allowed for a much higher catalytic activity. ${ }^{[9]}$ TEM images for PPP stabilized Pd-containing magnetite NPs presented in Figure S8 (SI) show similar morphology as that in the case of the PPQ NP stabilization (see Fig. S4, SI). Here, Pd NPs ( 3 nm) appear farther from the magnetite NPs. However, they are tethered to the latter via branched PPP molecules as was confirmed by TEM imaging (not shown) after magnetic separation. The XRD pattern of Pd-6 (Fig. S9, SI) contains typical reflections of magnetite and a full set of reflections of $\mathrm{Pd}^{0}{ }^{0.21]}$ XPS was not performed because polymer shielded the NP surface and no reliable signal could be obtained..$^{[9]}$

Table 2. Pd-containing magnetite NPs stabilized by PPP

\begin{tabular}{lll}
\hline Sample notation & $\begin{array}{l}\mathrm{Pd}(\mathrm{acac})_{2} \text { amount, } \\
(\mathrm{mmol})\end{array}$ & Pd NP size, nm \\
\hline Pd-4 & $0.0015(0.005)$ & $3.4 \pm 0.7$ \\
Pd-5 & $0.003(0.01)$ & $3.3 \pm 0.8$ \\
Pd-6 & $0.006(0.02)$ & $3.2 \pm 0.7$ \\
\hline
\end{tabular}

Catalytic properties of Pt- and Pd-containing magnetite NPs in furfural hydrogenation

To determine optimal reaction conditions of the FF hydrogenation, we varied the reaction temperature, pressure and the solvent type for the Pd-containing magnetite NPs prepared with $0.01 \mathrm{mmol}$ of $\mathrm{Pd}(\mathrm{acac})_{2}$ and stabilized by PPQ (Pd-1). The data obtained for the temperature and pressure dependences are presented in Figures S10 and S11 (SI), respectively. The FF conversion increases as the temperature and pressure increase, while the selectivity to FA goes through maxima at $120^{\circ} \mathrm{C}$ and $6 \mathrm{MPa}$. This can be explained by intensification of side reactions upon the increase of temperature and pressure. The identified side products are i-propyl-furfural ether, 2-furaldehyde diisopropyl acetal, tetrahydrofurfuryl alcohol, furan, tetrahydrofuran, and 2-methylfuran. ${ }^{[22]}$ Thus, the temperature of $120{ }^{\circ} \mathrm{C}$ and the pressure of $6 \mathrm{MPa}$ were chosen for all further experiments described in the paper.

It is well-established that the solvent type is an important factor in the FF hydrogenation. The solvent effect is typically associated with solvent polarity, solubility of hydrogen and FF, and interactions between the catalyst and the solvent. ${ }^{[22-23]}$ In this work, the influence of the solvent on the FF conversion and the selectivity to FA was studied for very polar solvents (water, methanol, ethanol), nonpolar solvents (hexane, toluene), and the solvent with medium polarity (i-propanol). It is noteworthy that the FF dielectric constant is $41.9,{ }^{[24]}$ while for PPQ, it is $2.8 .^{[25]}$ The FA dielectric constant was not found in literature but it should be comparable or even higher than that of FF. Figure 6 shows that the highest activity and selectivity are obtained in $i$-propanol.

There are several competing events (access to catalytic NP surface, adsorption of $F F$, etc.) which need to be taken into account when the solvent influence is discussed. Because the stabilizing polymer is quite hydrophobic, in a very polar solvent it will be compressed (precipitated) around Pd NPs, thus, preventing access of FF to the catalytic sites. Additionally, the solvent will compete with FF for adsorption on catalytic centers of NPs. Furthermore, when methanol and ethanol are used as solvents, acetals are formed as side products. ${ }^{[4,22 a, 26]}$ In the non-polar solvent PPQ will be swollen allowing for a complete access to the Pd NP surface, but the FF solubility will be limited, leading to a two-phase reaction solution. Moreover, the catalyst and FF will be in two different phases, thus limiting access to catalytic centers. On the other hand, in the solvent with medium polarity, $i$-propanol, an optimal balance of the FF solubility and satisfactory access to the metal catalytic centers is achieved leading to the highest activity and selectivity. In all further catalytic experiments, $i$-propanol was used as solvent.

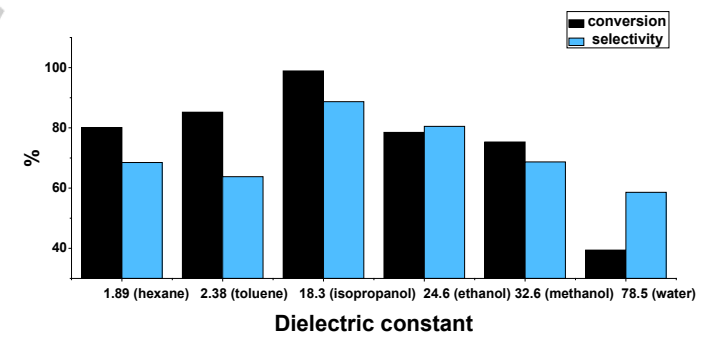

Figure 6. Dependences of the FF conversion and the selectivity to FA on the solvent dielectric constant. ${ }^{[27]}$

Table 3 shows catalytic data for the FF hydrogenation in the presence of Pt- and Pd-containing magnetite catalysts as well as the magnetite NPs stabilized by $\mathrm{PPQ}$ and PPP as control experiments.

The magnetite NPs alone stabilized by PPQ weakly catalyze FF hydrogenation resulting in the $15.3 \%$ conversion after $450 \mathrm{~min}$. However, FA is not detected. Apparently, FA formed immediately reacts with $i$-propanol leading to $i$-propyl-furfural ether. ${ }^{[1 \mathrm{~b}, 28]}$ Similar behavior was observed for $\mathrm{Fe}_{3} \mathrm{O}_{4}$-PPP, but the $\mathrm{FF}$ conversion is even lower: $2.7 \%$. The Pt-Fe $\mathrm{O}_{4} \mathrm{NPs}$ (Pt-2, Pt-3, and Pt-4) allow for nearly full conversion of FF after 450 min. (Pt-1 with the highest $\mathrm{Pt}$ loading was not studied because it contained Pt NPs which were 
not magnetically recoverable). It is noteworthy that both catalytic activity and selectivity improve with the decrease of the Pt content.

Table 3. FF conversion, product distribution, catalytic activity, and selectivity for Pt- and Pd-containing magnetite NPs in the FF hydrogenation. ${ }^{\text {[a] }}$

\begin{tabular}{|c|c|c|c|c|c|c|c|}
\hline \multirow{2}{*}{$\begin{array}{l}\text { Catalyst } \\
\text { notation }^{[b]}\end{array}$} & \multirow{2}{*}{$\begin{array}{l}\mathrm{Pt}(\mathrm{Pd}) \\
\text { amount, } \\
\text { wt.\% }\end{array}$} & \multirow[t]{2}{*}{ FF conversion, \% } & \multicolumn{3}{|c|}{ Products, \% } & \multirow{2}{*}{$\begin{array}{l}\text { Catalytic } \\
\min ^{-1}[\text { [e] }\end{array}$} & \multirow[t]{2}{*}{ Selectivity to FA, \% } \\
\hline & & & FA & Ether ${ }^{[c]}$ & Other ${ }^{[d]}$ & & \\
\hline $\mathrm{Fe}_{3} \mathrm{O}_{4}-\mathrm{PPQ}$ & - & 15.3 & - & 15.3 & traces & & - \\
\hline $\mathrm{Fe}_{3} \mathrm{O}_{4}-\mathrm{PPP}$ & - & 2.7 & - & 2.7 & traces & & \\
\hline Pt-2 & 0.9 & 98.7 & 72.8 & 25.1 & 0.8 & 20.1 & 73.8 \\
\hline Pt-3 & 0.5 & 98.9 & 74.3 & 23.4 & 1.2 & 50.2 & 75.1 \\
\hline Pt-4 & 0.3 & 98.8 & 78.5 & 19.6 & 0.7 & 85.1 & 79.5 \\
\hline $\mathrm{Pd}-1$ & 0.7 & 98.9 & 87.7 & 10.3 & 0.9 & 20.3 & 88.7 \\
\hline $\mathrm{Pd}-2$ & 1.2 & 97.8 & 84.8 & 11.8 & 1.2 & 12.6 & 85.2 \\
\hline $\mathrm{Pd}-3$ & 2.1 & 98.5 & 81.2 & 12.3 & 5.0 & 8.5 & 82.4 \\
\hline $\mathrm{Pd}-4$ & 0.3 & 98.8 & 98.1 & 0.7 & - & 871 & 99.3 \\
\hline $\begin{array}{l}\mathrm{Pd}-4 \text { third } \\
\text { use }\end{array}$ & 0.3 & 98.9 & 98.0 & 0.9 & & 865 & 99.1 \\
\hline $\begin{array}{l}\mathrm{Pd}-4 \quad \text { fifth } \\
\text { use }\end{array}$ & 0.3 & 98.6 & 97.8 & 0.8 & & 859 & 99.2 \\
\hline $\mathrm{Pd}-5$ & 0.8 & 99.7 & 99.1 & 0.6 & - & 234 & 99.4 \\
\hline $\mathrm{Pd}-6$ & 1.95 & 99.2 & 96.4 & 2.8 & - & 103 & 97.2 \\
\hline
\end{tabular}

[a] Reaction conditions: Temperature $120^{\circ} \mathrm{C}$, pressure $\left(\mathrm{H}_{2}\right) 6 \mathrm{MPa}$, furfural volume $2 \mathrm{~mL}$, $i$-propanol volume $48 \mathrm{~mL}$, reaction time 450 min; The catalyst amount was chosen to keep the molar ratio $\mathrm{Pt}(\mathrm{Pd}) /$ furfural equal $10^{-4} ;[\mathrm{b}] \mathrm{Pd}-4, \mathrm{Pd}-5$, and $\mathrm{Pd}-6$ were stabilized by $\mathrm{PPP}$, while all other catalysts are stabilized by PPQ; [c] i-propyl-furfural ether; [d] other: tetrahydrofurfuryl alcohol, 2-methylfuran, and tetrahydrofuran; [e] catalytic activity was calculated as moles of FF converted per mole of metal (Pt or Pd) per minute. 
The better catalytic performance at low catalytic metal loading could be due to elimination of steric shielding of NPs by each other when they are in close proximity. On the other hand, for $\mathrm{Pt}$ catalysts, the selectivity to $\mathrm{FA}$ at $\sim 99 \%$ conversion does not exceed $79.5 \%$ even at the lowest Pt loading (Pt-4, Table 3). For this catalyst, the activity calculated per the Pt mole reaches 85.1 $\mathrm{min}^{-1}$ which is comparable with the catalysts based on noble metals $(\mathrm{Pd}, \mathrm{Pt}, \mathrm{Ru})^{[4 \mathrm{e}, 26,29]}$ and by an order of magnitude higher than the activities of the $\mathrm{Ni}$ or $\mathrm{Cu}$ based catalysts. ${ }^{[1 \mathrm{~b}, 6,30]}$ For $\mathrm{Pt}$ NPs supported on inorganic oxides, high selectivity (up to $97 \%$ ) was observed but only at the conversion not exceeding $79 \% .{ }^{[4 \mathrm{e}]}$ In the case of Pd-containing magnetite NPs stabilized by PPQ, the selectivity to FA increased to $88.7 \%$ at the lowest Pd loading, but the activity was disappointedly low and did not exceed 20.3 $\mathrm{min}^{-1}$. Alternatively, for Pd-containing magnetite NPs stabilized by PPP, the high selectivity to FA of $99.3 \%$ at $\sim 99 \%$ conversion was achieved at a remarkable activity of $871 \mathrm{~min}^{-1}$.

It is noteworthy that the activity presented in Table 3 was calculated per mole of Pd. On the other hand, for NPs of $\sim 3 \mathrm{~nm}$, the fraction of surface atoms is only about $50 \%$ of all atoms, ${ }^{[31]}$ indicating that the turnover frequency (TOF) of this catalysts is at least $1742 \mathrm{~min}^{-1}$ if all the surface atoms are accessible for catalysis, indicating the lowest possible TOF. (Normally, only a fraction of surface atoms contains catalytic centers so actual TOF could be even higher.) This activity is much higher than that obtained for $\mathrm{Pd} / \mathrm{Al}_{2} \mathrm{O}_{3}$ (note that in the paper the TOF is given in $\left.\mathrm{h}^{-1}\right)^{[32]}$ and to the best of our knowledge, for all catalysts reported in literature. ${ }^{[22 b, 28 a, 32]}$ Moreover, in our case, it is combined with high conversion and selectivity.

It is worth noting that in all catalysts the sizes of Pd and Pt NPs are nearly the same $(\sim 3 \mathrm{~nm})$, allowing us to decouple the influence of the two other factors on catalysis: (i) type of a catalytic metal when the same stabilizing polymer is used or (ii) the type of a polymer for the same catalytic NPs. In the case of PPQ stabilization, $\mathrm{Pd}$ nanoparticles show higher selectivity at lower activity, while for Pt NPs the activity is higher, but only at low metal loading. A comparison of the catalytic activities of the Pdcontaining magnetite NPs stabilized by $\mathrm{PPQ}$ and PPP clearly demonstrates the advantages of the open and rigid structure of the hyperbranched PPP vs. linear PPQ. We believe that better access to the catalytic species in the PPP stabilized Pd-containing magnetite NPs governs their exceptional catalytic activity.

It is important to emphasize that PPP stabilization allows for not only much higher activity but also excellent selectivity, i.e., $99.3 \%$ vs. $88.7 \%$ for $P P Q$ stabilization at $99 \%$ conversion. This effect can be explained by tethering the Pd NPs to the $\mathrm{Fe}_{3} \mathrm{O}_{4} \mathrm{NPs}$ via a hyperbranched PPP chain instead of positioning the Pd NPs in the close proximity of the magnetite NPs. Indeed, as the control experiment showed, the magnetite NPs promote fast alcoholysis of FA to i-propyl-furfural ether. When magnetite NPs are well shielded by the polymer as in the case of PPP, this transformation is minimized leading to higher selectivity to FA.

To assess stability of the Pd-containing magnetite NPs capped by PPP, they were magnetically separated after the first catalytic reaction and tested in four consecutive catalytic experiments. The TEM image of the catalyst after the last reaction is presented in Figure $\mathrm{S} 12(\mathrm{SI})$. The catalyst looks virtually the same as the catalyst before the reaction (Fig. S8a, SI) owing to excellent stabilization by thermally stable, rigid PPP. The data presented in Table 3 demonstrate that both activity and selectivity are nicely preserved after five consecutive catalytic reactions, making these catalysts promising for future industrial applications.

\section{Conclusions}

Novel Pt- and Pd-containing magnetite NPs stabilized by linear PPQ or hyperbranched PPP have been synthesized and studied as catalysts in the FF hydrogenation to FA. These composite NPs were obtained in a one-pot procedure by thermal decomposition of the corresponding acetylacetonates in the reaction solution of preformed magnetite NPs. TEM shows formation of $\sim 3 \mathrm{~nm} \mathrm{NPs}$ with a darker electron contrast along with initial magnetite NPs. XRD and XPS confirm the magnetite structure and zero-valent character of $\mathrm{Pt}$ and $\mathrm{Pd}$ NPs. At comparatively low $\mathrm{Pt}$ and $\mathrm{Pd}$ precursor loadings the Pt or Pd NPs are located in the polymer shells of magnetic NPs, making them magnetically recoverable. Considering that catalytic NP sizes are nearly the same for the $P P Q$ and PPP stabilized catalysts, we were able to decouple the polymer structure influence on the catalytic performance. Hyperbranched PPP allows for better access to catalytic species compared to linear $P P Q$, leading to much higher catalytic activity. A larger distance between $\mathrm{Pd}$ and $\mathrm{Fe}_{3} \mathrm{O}_{4} \mathrm{NPs}$ in the case of PPP tethering also allows for higher selectivity to FA $(99.3 \%)$ at nearly $100 \%$ conversion. The combination of high performance with increased stability and easy magnetic recovery makes these catalysts promising for further applications in fuel and energy related fields.

\section{Supporting Information}

Information about experimental procedures, methods, magnetic separation, TEM images, XRD patterns, XPS data and dependences of the FF conversion and the selectivity to FA on temperature and pressure are provided in the Supporting Information.

\section{Acknowledgements}

The research leading to these results has received funding from the European Community's Seventh Framework Programme [FP7/2007-2013] under grant agreement no. 604296. Z.S., E.S., and N.K. thank Russian Foundation for Basic Research (projects 17-03-00578 and 16-53-45037) for funding. E.S. and V.M. thank Russian Foundation for Basic Research (projects 17-08-00660 and 15-08-00305) for funding. V.M., L.M., and E.S. thank Russian Science Foundation (grant 17-19-01408) for funding. V.M. also thanks the Ministry of Education and Science of the Russian Federation for the financial support. The authors thank the Indiana University Nanoscale Characterization Facility for access to the 
instrumentation as well as NSF grant \#CHE-1048613 which funded Empyrean from PANalytical.

Keywords: biooil • furfuryl alcohol • hyperbranched polymer • magnetically recoverable catalysts

[1] a) M. Hronec, K. Fulajtarova, I. Vavra, T. Sotak, E. Dobrocka, M Micusik, Appl. Catal., B 2016, 181, 210-219; b) S. A. Khromova, M. V. Bykova, O. A. Bulavchenko, D. Y. Ermakov, A. A. Saraev, V. V. Kaichev, R. H. Venderbosch, V. A. Yakovlev, Top. Catal. 2016, 59, 1413-1423.

[2] D. M. Alonso, J. Q. Bond, J. A. Dumesic, Green Chem. 2010, 12, 14931513.

[3] a) H. C. Anderson, in Secondary, 1977; b) J.-M. Pin, N. Guigo, A. Mija, L. Vincent, N. Sbirrazzuoli, J. C. van der Waal, E. de Jong, ACS Sustain. Chem. Eng. 2014, 2, 2182-2190.

[4] a) X. Chen, L. Zhang, B. Zhang, X. Guo, X. Mu, Sci. Rep. 2016, 6 , 28558; b) F. Li, B. Cao, R. Ma, J. Liang, H. Song, H. Song, Can. J. Chem. Eng. 2016, 94, 1368-1374; c) R. V. Maligal-Ganesh, C. Xiao, T. W. Goh, L.-L. Wang, J. Gustafson, Y. Q. Pei, Zhiyuan; Johnson, D. D.; S. Zhang, F. Tao, W. Huang, ACS Catal. 2016, 6, 1754-1763; d) O. F. Aldosari, S. Iqbal, P. J. Miedziak, G. L. Brett, D. R. Jones, X. Liu, J. K. Edwards, D. J. Morgan, D. K. Knight, G. J. Hutchings, Catal. Sci. Technol. 2016, 6, 234-242; e) M. J. Taylor, L. J. Durndell, M. A. Isaacs, C. M. A. Parlett, K. Wilson, A. F. Lee, G. Kyriakou, Appl. Catal., B 2016, $180,580-585$; f) W. Gong, C. Chen, Y. Zhang, H. Zhou, H. Wang, H. Zhang, Y. Zhang, G. Wang, H. Zhao, ACS Sustain. Chem. Eng. 2017, 5, 2172-2180

[5] a) Y. Zhu, S. C. Peng, A. Emi, Z. Su, Monalisa, R. A. Kemp, Adv. Synth. Catal. 2007, 349, 1917-1922; b) V. Polshettiwar, R. Luque, A. Fihri, H. Zhu, M. Bouhrara, J.-M. Basset, Chem. Rev. 2011, 111, 3036-3075; c) L. M. Rossi, F. P. Silva, L. L. R. Vono, P. K. Kiyohara, E. L. Duarte, R. Itri, R. Landers, G. Machado, Green Chem. 2007, 9, 379-385; d) A. Saha, J. Leazer, R. S. Varma, Green Chem. 2012, 14, 67-71; e) B. R. Vaddula, A. Saha, J. Leazer, R. S. Varma, Green Chem. 2012, 14 2133-2136; f) D. Wang, D. Astruc, Chem. Rev. 2014, 114, 6949-6985; g) A.-H. Lu, E. L. Salabas, F. Schueth, Angew. Chem. Int. Ed. 2007, 46, $1222-1244$; h) A. Bazgir, G. Hosseini, R. Ghahremanzadeh, ACS Comb. Sci. 2013, 15, 530-534; i) D. Kundu, T. Chatterjee, B. C. Ranu, Adv. Synth. Catal. 2013, 355, 2285-2296.

[6] A. Halilu, T. H. Ali, A. Y. Atta, P. Sudarsanam, S. K. Bhargava, S. B. Abd Hamid, Energy Fuels 2016, 30, 2216-2226.

[7] R. K. Singh, R. M. Mehta, R. G. Bass, High Perform. Polym. 1995, 7.

[8] N. V. Kuchkina, M. S. Zinatullina, E. S. Serkova, P. S. Vlasov, A. S. Peregudov, Z. B. Shifrina, RSC Adv. 2015, 5, 99510-99516.

[9] N. Baird, J. W. Dittmar, Y. B. Losovyj, D. G. Morgan, B. D. Stein, M. Pink, N. V. Kuchkina, E. S. Serkova, O. L. Lependina, M. E. Grigoriev, A
I. Sidorov, M. G. Sulman, Z. B. Shifrina, L. M. Bronstein, ACS Appl. Mater. Interfaces 2017, 9, 2285-2294.

[10] N. Baird, Y. Losovyj, E. Y. Yuzik-Klimova, N. V. Kuchkina, Z. B. Shifrina, M. Pink, B. D. Stein, D. G. Morgan, T. Wang, M. Rubin, A. Sidorov, E. M Sulman, L. M. Bronstein, ACS Appl. Mater. Interfaces 2016, 8, 891-899.

[11] a) Y. Tian, B. Yu, X. Li, K. Li, J. Mater. Chem. 2011, 21, 2476-2481; b) L. W. Finger, R. M. Hazen, A. M. Hofmeister, Phys. Chem. Miner. 1986, 13 215-220.

[12] V. M. Goldschmidt, J. Iron Steel Inst. 1947, 155, 221-224.

[13] a) D. D. Hawn, B. M. DeKoven, Surf. Interface Anal. 1987, 10, 63-74; b) M. Muhler, R. Schoegl, G. Ertl, J. Catal. 1992, 138, 413-444; c) T. Yamashita, P. Hayes, Appl. Surf. Sci. 2008, 254, 2441-2449.

[14] T. Fujii, F. M. F. de Groot, G. A. Sawatzky, F. C. Voogt, T. Hibma, K. Okada, Phys. Rev. B. 1999, 59, 3195-3202.

[15] R. Harpeness, A. Gedanken, J. Mater. Chem. 2005, 15, 698-702.

[16] K. E. Gilbert, J. J. Gajewski, J. Org. Chem. 1982, 47, 4899-4902.

[17] F. Cui, L. Dou, Q. Yang, Y. Yu, Z. Niu, Y. Sun, H. Liu, A. Dehestani, K Schierle-Arndt, P. Yang, J. Am. Chem. Soc. 2017, 139, 3027-3032.

[18] Z.-j. Wang, Y. Xie, C.-j. Liu, J. Phys. Chem. C 2008, 112, 19818-19824.

[19] R. Wojcieszak, M. J. Genet, P. Eloy, P. Ruiz, E. M. Gaigneaux, J. Phys. Chem. C 2010, 114, 16677-16684.

[20] F. Liao, T. W. B. Lo, D. Sexton, J. Qu, C.-T. Wu, S. C. E. Tsang, Catal Sci. Technol. 2015, 5, 887-896.

[21] G. Bredig, R. Allolio, Z. physik. Chem. 1927, 126, 41-71.

[22] a) B. Chen, F. Li, Z. Huang, G. Yuan, Appl. Catal., A 2015, 500, 23-29; b) A. Fuente-Hernández, R. Lee, N. Beland, I. Zamboni, J.-M. Lavoie, Energies 2017, 10, 286

[23] a) B. S. Akpa, C. D'Agostino, L. F. Gladden, K. Hindle, H. Manyar, J. McGregor, R. Li, M. Neurock, N. Sinha, E. H. Stitt, D. Weber, J. A. Zeitler, D. W. Rooney, J. Catal. 2012, 289, 30-41; b) N. M. Bertero, A. F. Trasarti, C. R. Apesteguia, A. J. Marchi, Appl. Catal., A 2011, 394, 228238; c) P. G. N. Mertens, F. Cuypers, P. Vandezande, X. Ye, F. Verpoort, I. F. J. Vankelecom, D. E. De Vos, Appl. Catal., A 2007, 325 130-139.

[24] P. H. Fang, S. Marzullo, W. S. Brower, Phys. Rev. 1957, 108, 242-243

[25] B. Sillion, G. Rabilloud, J. Garapon, O. Gain, J. Vallet, Mater. Res. Soc. Symp. Proceed 1995, 381, 93-104.

[26] A. O'Driscoll, J. J. Leahy, T. Curtin, Catal. Today 2017, 279, 194-201.

[27] "Common Organic Solvents: Table of Properties" to be found under https://www.organicdivision.org/orig/organic solvents.html, 2017.

[28] a) A. B. Merlo, V. Vetere, J. F. Ruggera, M. L. Casella, Catal. Commun. 2009, 10, 1665-1669; b) J. Li, J.-I. Liu, H.-j. Zhou, Y. Fu, ChemSusChem, 9, 1339-1347.

[29] K. Fulajtarova, T. Sotak, M. Hronec, I. Vavra, E. Dobrocka, M. Omastova, Appl. Catal., A 2015, 502, 78-85

[30] C. Sun, P. Zeng, M. He, X. He, X. Xie, Catal. Commun. 2016, 86, 5-8.

[31] E. Roduner, Chem. Soc. Rev. 2006, 35, 583-592.

[32] S. Bhogeswararao, D. Srinivas, J. Catal. 2015, 327, 65-77. 


\section{Entry for the Table of Contents}

\section{FULL PAPER}

\section{Catalyst for biooil component} hydrogenation: Stabilization of composite $\mathrm{Pd}-\mathrm{Fe}_{3} \mathrm{O}_{4}$ nanoparticles by rigid hyperbranched pyridylphenylene polymer allows for remarkable activity and high selectivity in the furfuryl alcohol synthesis by hydrogenation of the major biooil ingredient, furfural. The magnetic component of the catalyst allows for easy magnetic separation. The catalyst demonstrated high stability in repeated use.

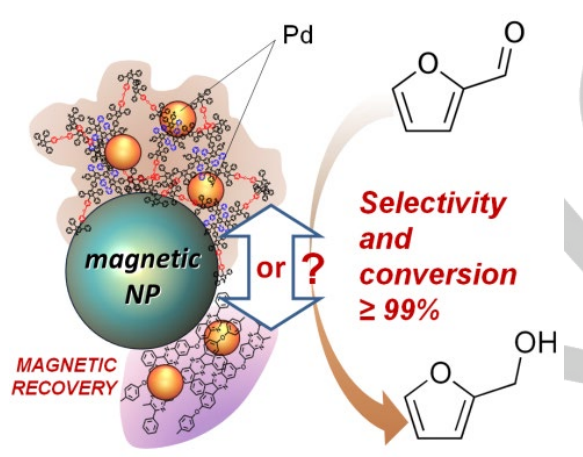

K. Alibegovic, D. G. Morgan, Y. Losovyj, M. Pink, B. D. Stein, N. V. Kuchkina, E.

S. Serkova, K. E. Salnikova, Z. B.

Shifrina, V. G. Matveeva, E. M. Sulman,

L. M. Bronstein

Page No. - Page No.

Efficient Furfuryl Alcohol Synthesis from Furfural over Magnetically Recoverable Catalysts: Does the Catalyst Stabilizing Medium Matter? 
